\title{
The burden of head and neck cancer in Africa: the status quo and research prospects
}

\author{
SADJ September 2018, Vol. 73 No. 8 p477 - p488
}

HA Adeola*, AH Afrogheh", JJ Hille ${ }^{+}$

\section{ABSTRACT}

\section{Background}

The increasing incidence of head and neck cancer (HNC) in Africa is mostly due to increasing smoking habits, westernization in lifestyle and diet, HIV/HPV and unfavourable fiscal policies for health.

Oral cancer potentially constitutes a notable fraction of the global cancer burden, particularly in developing Africa. A heterogeneity in the anatomy-based HNC nomenclature has resulted in underestimation of its incidence and significance worldwide.

\section{Method}

The global cancer statistics database (GLOBOCAN) was used to evaluate the combined data of cancer incidence in two subsets: a.) excluding $\mathrm{HNC}$ and b.) including oesophageal cancers (Upper Aerodigestive Tract Cancer: UADTC). Combined age standardized incidence rates (ASIR) of HNC and UADTC were analyzed using both regional and individual country-derived data.

\section{Result}

Frequency ranking of $\mathrm{HNC}$ in Africa increases dramatically when cancers from contiguous $\mathrm{HN}$ regions are evaluated, compared with considering oral cancer data alone.

UADTC and HNC ranked second and third in Africa, respectively. Similar trends were observed by global development index evaluation.

\section{Author affiliations:}

* Henry A. Adeola ${ }^{1,2} B D S, P h D(M e d)$.

\# Amir H. Afrogheh ${ }^{1,3}$ BDS, FCPath (SA).

+ Johannes J. Hille ${ }^{1,3}$ DDS, M Dent, FCPath (SA).

'Department of Oral and Maxillofacial Pathology, Faculty of Dentistry, University of the Western Cape at Tygerberg Hospital, Cape Town, South Africa.

${ }^{2}$ Division of Dermatology - Hair and Skin Laboratory, Department of Medicine, Faculty of Health Sciences and Groote Schuur Hospital, University of Cape Town, Cape Town, South Africa.

${ }^{3}$ National Health Laboratory Service (NHLS), Tygerberg, South Africa Corresponding author: Henry A. Adeola

Department of Oral and Maxillofacial Pathology, Faculty of Dentistry,

University of the Western Cape at Tygerberg Hospital, Cape Town,

South Africa. Division of Dermatology - Hair and Skin Laboratory,

Department of Medicine, Faculty of Health Sciences and Groote Schuur Hospital, University of Cape Town, Cape Town, South Africa.

Tel: +27 (0)21650 3729

E-mail: henry.adeola@uct.ac.za
ACRONYMS
ASIR: $\quad$ Age Standardized Incidence Rates
ASMR: $\quad$ Age Standardized Mortality Rate
GLOBOCAN: Global Cancer Statistics Database
HNC: $\quad H e a d$ And Neck Cancer
IARC/WHO: International Agency For Research On
Cancer/World Health Organisation
LMIC: $\quad$ Low And Middle Income Countries
NCDs: Non-Communicable Diseases
UADTC: $\quad$ Upper Aerodigestive Tract Cancer

\section{Conclusion}

With the exclusion of a few urban regions, the diagnostic and treatment opportunities for HN/UADT cancers in sub-Saharan Africa are dismal.

Effective education and research efforts by local and international (non-) governmental agencies are urgently required to reduce the associated morbidities.

\section{Keywords}

Oral cancer burden, Africa, research, HNC, UADTC

\section{INTRODUCTION}

Cancer is a major public health problem and a leading cause of death worldwide. It was estimated that between 1975 and 1990, there was a 37\% increase in cancer cases with an annual growth rate of $2.1 \%$. By the mid-nineties, over 10 million new cases were diagnosed with up to 6 million reported deaths. It is expected that this figure would have doubled by 2020. ${ }^{1}$ Similar trends were observed in the GLOBOCAN 2008 database where approximately $60 \%$ mortality to incidence ratio was observed.

It was also observed that $56 \%$ and $64 \%$ incidence and mortality figures respectively were reported in the developing world where cancer prognosis tends to be worse, due to late stage presentation, limited human resources and poorer infrastructure. ${ }^{2}$ Most recently, global cancer incidence and mortality figures were reported to be 14 and 8.3 million respectively, ${ }^{3-5}$ showing a steady increase as earlier predicted.

Oral cancer, which can be described as a malignant neoplasia originating in the oral cavity or on the lip, ${ }^{6}$ ranks as the sixth most common cancer worldwide and as third in developing nations, with a high age standardized 
mortality rate (ASMR) of 6.8 (per 100,000 persons per year) in Black men as compared with their White or Hispanic counterparts. ${ }^{7-9}$

However, there is apparent under-reporting of cases in many countries in Africa due to lack of cancer registries, cancer control programmes, modern health infrastructure, access to healthcare, finances, educational levels and existing religious and cultural beliefs. In addition, most databases have considered cancer in various anatomical locations as different entities attracting separate consideration, albeit that the processes of squamous carcinogenesis in the UADT are biologically similar.

This pattern is also extended to cancers in the anatomically contiguous head and neck region and has resulted in ambiguity in nomenclature. For example, terminologies like 'oral cancer", "orofacial cancer", "oropharyngeal cancer", "head and neck cancer", and "upper aerodigestive cancer" have been used interchangeably to describe mainly malignant squamous tumours in the maxillofacial region. To be able to address these inconsistencies in anatomybased nomenclature and to standardize the comparison of epidemiologic studies, it has been suggested that the rubrics of the International Classification of Disease (ICD-10) for oncology should be employed for cancers in the head and neck region. ${ }^{10}$

The inherent heterogeneity in oral cancers and the potential direct extension of these lesions depending on the time of presentation deserve careful consideration, particularly in Africa where most patients present late. Besides, due to limited manpower most cancer cases involving the lip and oral cavity, nasopharynx, other pharynx, larynx, and upper oesophagus may be diagnosed by the oral and maxillofacial pathologist or general pathologist in most African countries.

Presenting the burden of oral cancer in a fragmented manner may not reflect its high importance. There is an urgent need for coordinated research amongst health governing bodies and policy makers especially in Africa where most cases are not accurately reported.

Low and middle income countries (LMIC) account for 80\% of the worldwide cancer burden, but receive only $5 \%$ of the global financial resources for cancer care. ${ }^{11}$ Many countries in Africa are undergoing economic modernization resulting in major changes in diet and lifestyle, smoking, and in sexual behaviour, having a potential influence on infections such as Human Immunodeficiency Virus (HIV) and Human Papillomavirus (HPV).

The burden of infectious diseases like malaria, cholera, HIV and tuberculosis (TB) has been a focus in Africa, against a backdrop of an increasing burden of non-communicable diseases (NCDs) such as cancer. ${ }^{12}$ According to the World Health Organization (http://www.who.int) NCD factsheet in 2011, NCD-related mortality by 2030 will exceed the combined mortality of maternal and perinatal deaths from communicable and nutritional diseases.

The biologic behaviour of oral cancers vis-à-vis determining which would run an aggressive or indolent course, adds another layer of intricacy to diagnosis and management. ${ }^{13}$
This paper, using a quantitative/descriptive analysis of the IARC/WHO GLOBOCAN database (http://globocan. iarc.fr), demonstrates that the combined burden of oral cancer in Africa is much higher than is often reported. This emphasizes the urgent need for further research into the true burden in the African continent.

\section{MATERIALS AND METHODS}

We reviewed secondary data archived by $\mathrm{WHO}$ and IARC in GLOBOCAN $2008^{14}$ and $2012^{5}$ global cancer statistics.

Age standardized incidence rates for cancers affecting different regions of the human body were documented and analyzed using quantitative statistical methods; and visualized using stacked bar graphs, pie charts and tables.

\subsection{Database and data mining}

The GLOBOCAN database available at IARC provides updates of age-standardized incidence, prevalence and mortality rates of major cancer types for 184 nations of the world. ${ }^{5}$ The data are available for both sexes. There is a continual effort to improve the quality of data and coverage, albeit figures may not be consistent over a prolonged period and may result in differences every update.

Hence the database may not be optimal for making time-course statistical inferences due to potential methodological differences between updates. Data are derived from national and regional sources using percentage data coverage, rates and frequencies. The mode of estimation is country-specific and the quality of data is dependent on the standard of information available locally.

Estimates are made where local sources are inadequate. Country-specific summaries of the burden of disease of any major type of cancer can be accessed using the "factsheet" option. Typically, this option generates basic statistical summaries about the eight commonest cancers as well as regional and country-specific data. In addition, an option for simple online analysis can generate additional visualization such as maps and graphs.

\subsection{Combined incidences analysis}

ASIR for contiguous regions of the oral and maxillofacial and head and neck structures were combined. The newly generated combined incidence was compared with the incidences of other major cancer types in the body, excluding non-melanoma skin cancer.

This was done for sixteen representative regions of the world as well as twelve specific countries which were selected to reflect the comparative differences between Africa and other regions of the world.

\subsubsection{Head and Neck Cancer (HNC)}

ASIR for cancers involving the lip and oral cavity, nasopharynx, other pharynx, and larynx (which are typically diagnosed by many oral and maxillofacial pathologists in the African diagnostic histopathologic settings) were combined to reveal the true burden of Head and Neck cancers in Africa (Figure 1, p479). 


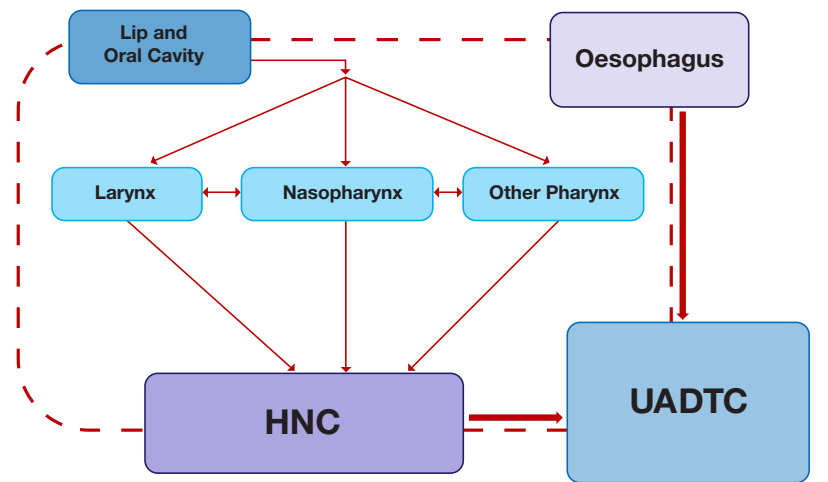

Figure 1. Workflow for combined ASIR of HNC and UADTC in Africa. Lip and oral cavity, nasopharynx, larynx and other pharynx were combined to get the HNC subset. Addition of esophagus to HNC results in the formation of the UADTC subset. As the combined ASIR of the constituent regions are added together, the burden grows.

The combined ASIR of these four contiguous regions was termed "Head and Neck Cancer (HNC)". ${ }^{15-17}$ A HNC burden map was also constructed using the online analysis function.

\subsubsection{Upper Aerodigestive Tract Cancer (UADTC)}

Considering that oral and maxillofacial pathologists sometimes diagnose oesophageal pathological lesions as well, a second subset was created which included cancerous lesions of the oesophagus.

The five regions included in this subset were lip and oral cavity, nasopharynx, other pharynx, larynx and esophagus (Figure 1, above).

This subset was termed "Upper Aerodigestive Tract Cancer (UADTC)". ${ }^{18-21}$ The online analysis function was used to construct a UADTC burden map.

\subsection{Statistical analysis}

Data was extracted from the GLOBOCAN website and saved in spreadsheets (Microsoft Excel version 2010).

HNC and UADTC combinations were performed in separate worksheets. For regional and country-specific analysis, pie charts and stacked bar graphs were constructed using the same version of Microsoft Excel.

The combined ASIR of HNC and UADTC in African regions and countries were compared with those of other more developed parts of the world. The proportions of the burden of HNC and UADTC compared with all other cancers were evaluated using stacked bar graphs.

\subsubsection{Regional ranking}

Sixteen regions were examined to assess how the combined ASIR of HNC and UADTC performed globally. Continental regions $(n=4)$ included were: Africa, Europe, North America and Asia.

Subcontinental regions $(n=8)$ included were: Sub-Saharan Africa, Western Africa, Eastern Africa, Southern Africa, Northern Europe, Western Europe, Southern Europe and Central and Eastern Europe.
Other regions $(n=4)$ included were: the European Union (EU-27), World, More-developed regions and Less-developed regions of the world. The Emerging HNC and UADTC rankings for these regions were evaluated for comparison.

\subsubsection{Country-specific ranking}

Ranking of HNC and UADTC was also examined at representative but randomly selected sub-Saharan and non-African country levels. This was done by comparing $\mathrm{HNC}$ and UADTC burden in the twelve selected countries.

African countries $(n=6)$ included were: the Republic of South African Republic (South Africa), Mozambique, Zambia, Kenya, Nigeria and Sudan. Other countries $(n=6)$ included were: the United States of America (USA), Japan, Germany, United Kingdom (UK), France (La Reunion) and France (Metropolitan).

HNC and UADTC burdens for these regions were compared with the data for all other cancers as had been effected for the regions. These burdens were also compared between African countries and the other six countries which had been included.

Finally, we compared the GLOBOCAN 2012 with the 2008 data using a similar methodology. Briefly, HNC and UADTC burdens were evaluated and compared for African regions and countries and other developed regions of the world. Also, the combined ASIRs of HNC and UADTC were compared with all other cancers.

\section{RESULTS}

The precise burden of cancers in the head and neck regions in Africa has been unclear due to several mitigating factors such as poor documentation of cases, poorly defined nomenclature and a lack of well-established functioning cancer registries across the continent.

Our findings using this uncomplicated approach to redistribute the combined Head and Neck cancer ASIR data have spurred our interest in uncovering the true burden of cancer in Africa and in enhancing possible understanding of the economic and health impact on the populations.

Currently, there is a dearth of locally derived data on head and neck cancer in many African nations; and it is plausible that accurate determination of the burden of a disease can be extrapolated from global data repositories as has been done here. All Age-Standardized Incidence Rates (ASIR) values are "per 100,000 persons per year".

\subsection{Global HNC and UADTC distribution}

The combined indices of head and neck cancers, HNC and UADTC, were compared worldwide to determine the burden of disease in Africa compared with the rest of the word. For HNC, estimated ASIR were combined for the nasopharynx, other pharynx, lip, oral cavity and larynx.

Similarly, ASIR for these regions including oesophagus were combined for UADTC. ASIR distribution for HNC revealed that less than $25 \%$ percent of the Africa continent, 
mainly in the central and western parts of northern Africa have ASIR of less than 6.2, while other regions recorded greater values (Figure 2A, p481).

Hot spots for HNC as observed in this estimation were identified in the southern African region where ASIR values were mostly greater than 13.1 , whilst the values were greater than 20.0 in the north-western parts of this region and in Madagascar. Values greater than 13.1 but less than 20.0 were found as well in some parts of eastern Africa.

In comparison with the rest of the world, hot spots for HNC were identified in northern and eastern Europe and most of the northern, central and southern parts of Asia. No region in northern America has HNC ASIR values greater than 20.0 except in the Caribbean region; and more than 50\% of the northern America region has an HNC ASIR value below 13.1.

This picture changes dramatically when we look at the ASIR's of the UADTC; almost $25 \%$ of Africa is greater than 20.0. Nearly half of Africa is a UADTC hot spot if we lower the ASIR cut-off values to 14.2 and above.

There are areas with ASIR greater than 28.0 and less than 61.4 in eastern, southern and south-eastern Africa, as well as Madagascar (Figure 2B, p481). The UADTC burden did not seem to change much in northern America, while there was a shift in high burden of UADTC towards the southern regions of Asia.

\subsection{Regional and Country derived data}

We have chosen a few countries to demonstrate the difference in burden between highly developed nations compared with the less developed world. Twelve countries and sixteen regions were sampled in this study.

\subsubsection{Combined ASIR Ranking}

The proportion of combined incidence of HNC and UADTC compared with that of other cancers was evaluated and ranked at the regional and country level. With a combined ASIR of 7.1, HNC ranked sixth in the world, in keeping with the literature. However, UADTC ranked 3rd globally with a combined ASIR of 12.0 .

The ranking ranged between the fourth and fifth positions for most European regions and for northern America. HNC ranked fifth in Asia with a combined ASIR of 8.0 and notably was second in the UADTC ranking (Table 1, p486). The picture was quite different when we considered African regions which generally had rankings of higher incidence both for HNC and UADTC. HNC ranked third in Africa with a combined ASIR of 7.8, while UADTC ranked first with a combined ASIR of 13.2 .

Looking at the sub-Saharan region HNC ranked fourth while UADTC ranked second. HNC ranked fourth and third in southern and western Africa respectively; while UADTC ranked second and third in these regions respectively.

Interestingly, UADTC ranked first in eastern Africa with a combined ASIR of 17.9 with a HNC ranking of fourth position and combined ASIR of 7.5.
These increasing trends of ranking in Africa were also demonstrated by stacked bar graphs and pie charts. (Figure 3A, p482 and Figure 4, p483).

High ranking of HNC and UADTC continues to emerge even in country-derived data (Figure 3B, p482 and Figure 5, p484).

We observed that HNC ranked second, third, fourth and fifth for Sudan, Nigeria, Zambia, South Africa and Mozambique respectively; UADTC ranked first, third, third, second and third in these five African countries respectively.

Low rankings were found in Japan, the United States of America and Germany where HNC rankings were ninth, sixth and fifth respectively; and their corresponding UADTC rankings were all fifth respectively (Table 2, p486)).

HNC ranked fifth in the United Kingdom while UADTC ranked in fourth position. In France, HNC ranked second and fourth in La Reunion and the Metropolitan regions respectively. UADTC ranked first in La Reunion region but in contrast remains fourth in the Metropolitan areas.

This shift in ranking between the urban and rural areas both regionally and by country seems to indicate that the burden of oral cancer requires further investigation.

\subsubsection{Comparisons according to Human Development Index}

Another indicator that we examined was to compare the GLOBOCAN data on more developed regions of the world with that of less developed regions. Usefully, the database has readymade estimates on these two regions. Not surprisingly, when computing the HNC and UADTC ASIRs we found high rankings in less developed regions compared with the more developed region, a finding consistent with our findings so far.

HNC ranked fourth in the the less developed world with a combined ASIR of 8.4; while UADTC ranked second in relation to other cancer with a combined ASIR of 15.6. In contrast, HNC ranked sixth in the more developed world with a combined ASIR of 3.6 and UADTC, with a combined ASIR of 5.1, ranking fourth (Table 1, P486). Similar comparisons of ranking between these two regions can also be seen in the stacked bar graphs and pie charts (Figures 3A, P482 and Figure 5, p484).

\subsubsection{Consistent trends in 2012 update to the GLOBOCAN 2008 Database}

While we had observed these pertinent findings using the GLOBOCAN 2008 database, we then evaluated the latest database for any major differences in HNC and UADTC and found that not much has changed in terms of these combined burdens.

Notably, UADTC now ranks second in Africa with a combined ASIR of 11.7; but still ranks fifth in North America with a combined ASIR of 6.2. HNC retains its third position ranking in Africa with a combined ASIR of 7.3 but drops to sixth position in North America with a combined ASIR of 4.6 (Figure 6, p484). 


\section{A HNC}

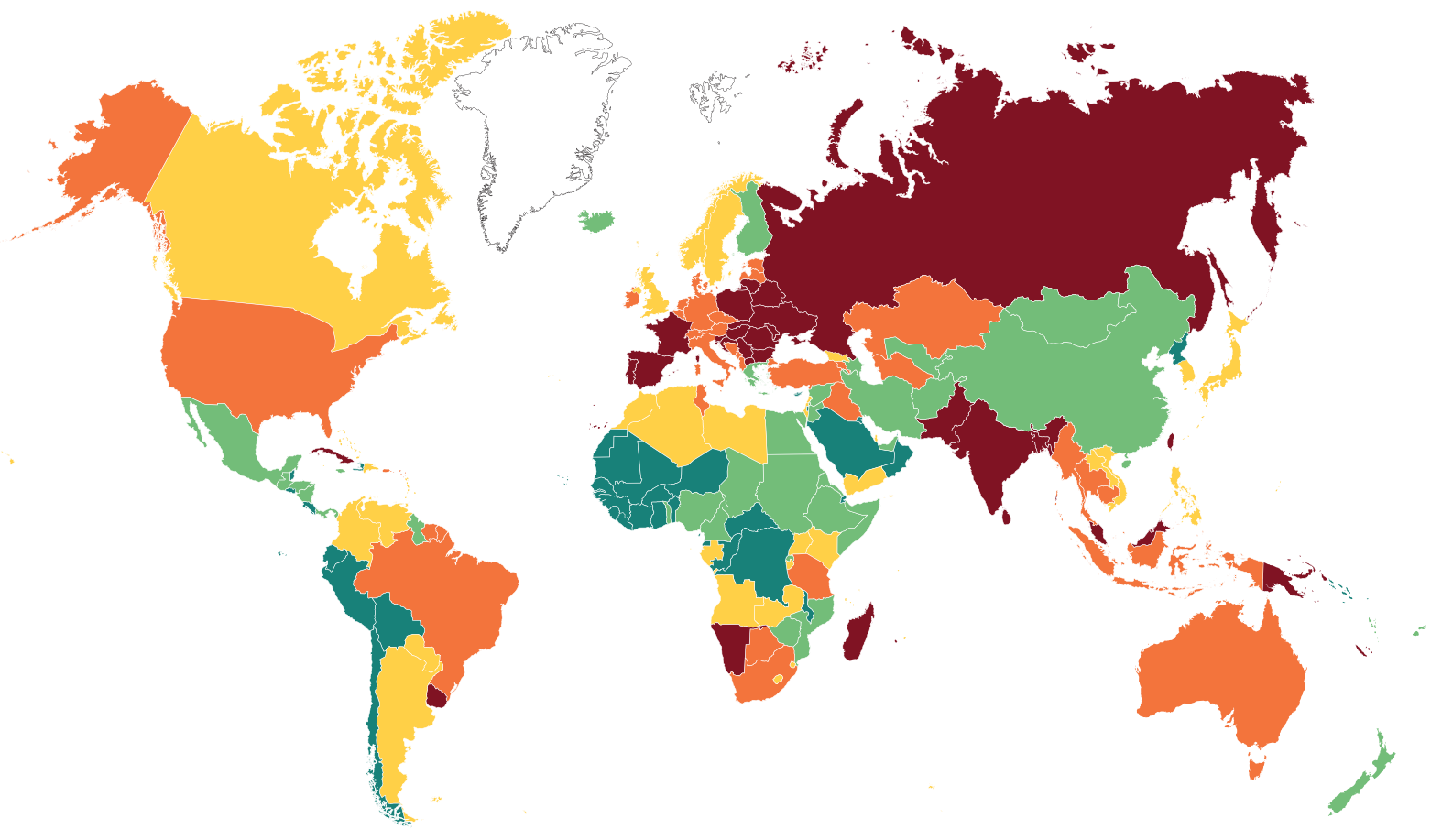

$\square<6.2 \square<8.8 \square<13.1 \square<20.0 \square<49.8$

\section{B UADTC}

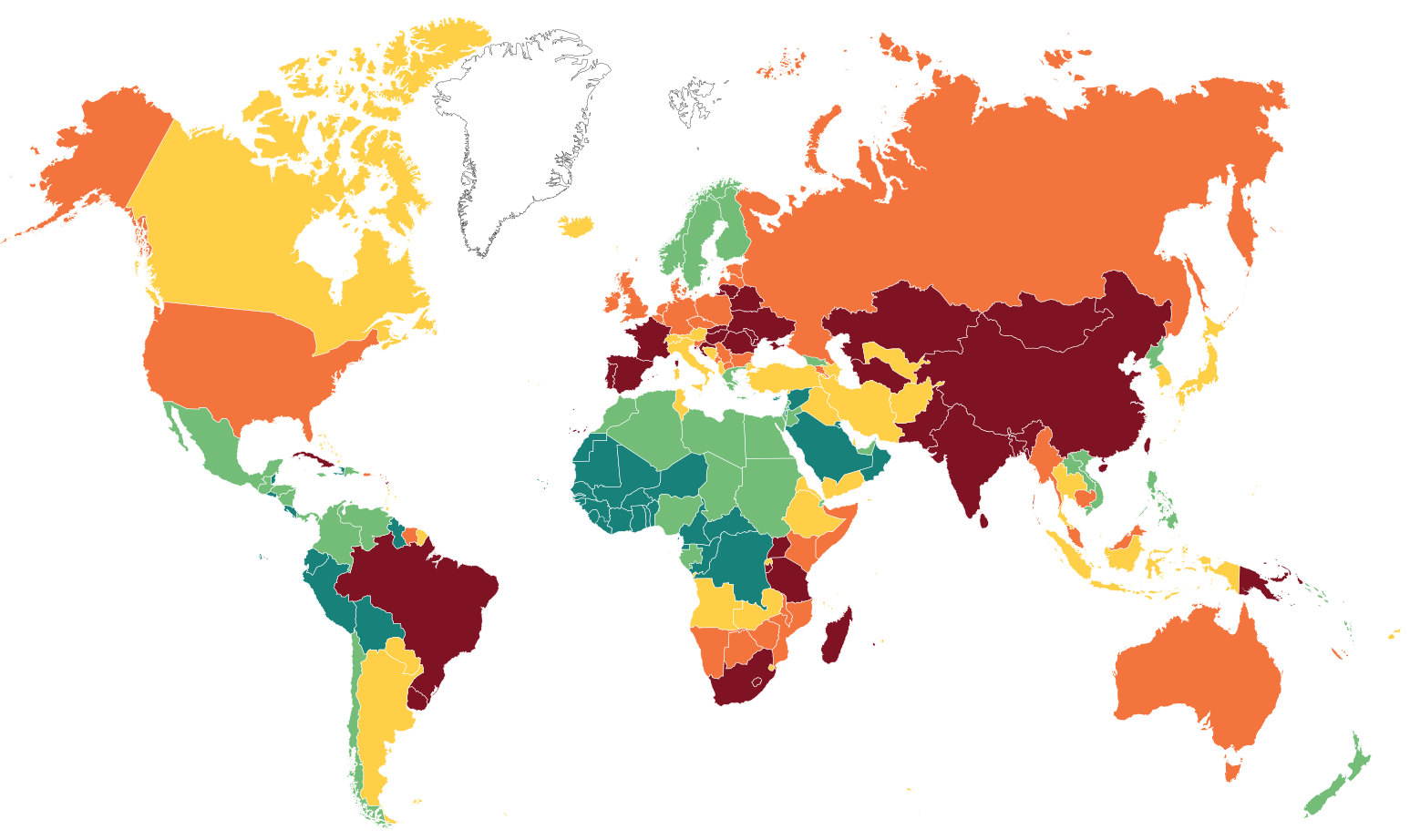

$\square<8.4 \square<14.2 \square<20.1 \square<28.0 \square<61.4$

Figure 2. Map of global distribution of combined ASIR of oral cancer (A) Shows the Age standardized incidence rate (ASIR) of HNC is high in many regions of Africa as compared with the rest of the world. (B) Shows an increased burden of oral cancer when esophageal cancers are added to form the UADTC subset. Nearly half of the continent had hot spots with ASIR values of greater than 14.1. This demonstrates that the burden of oral cancer may be higher than currently reported in many literatures. There are areas of very high ASIRs in the southern and eastern regions of Africa as shown here. 


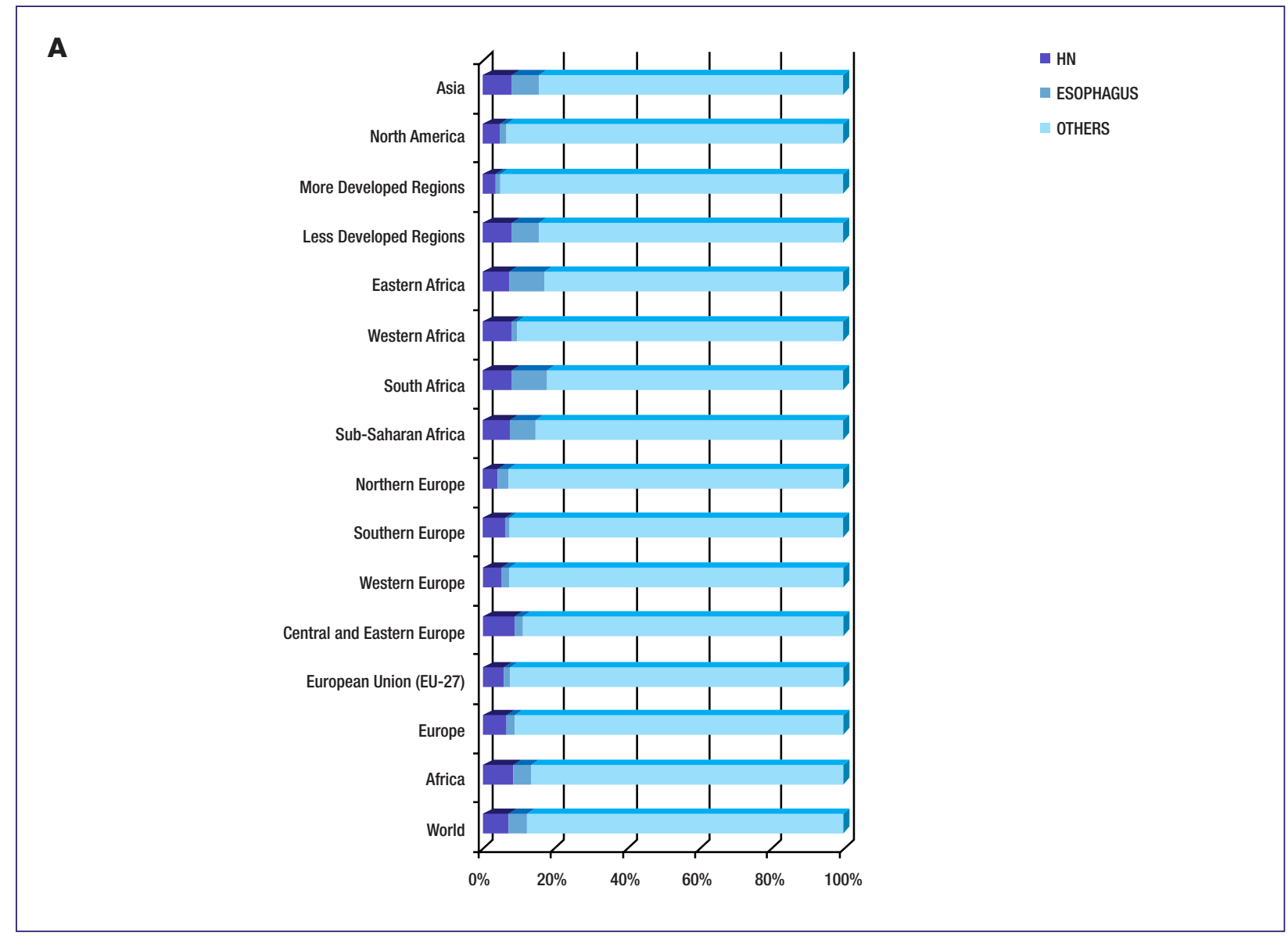

\section{B}

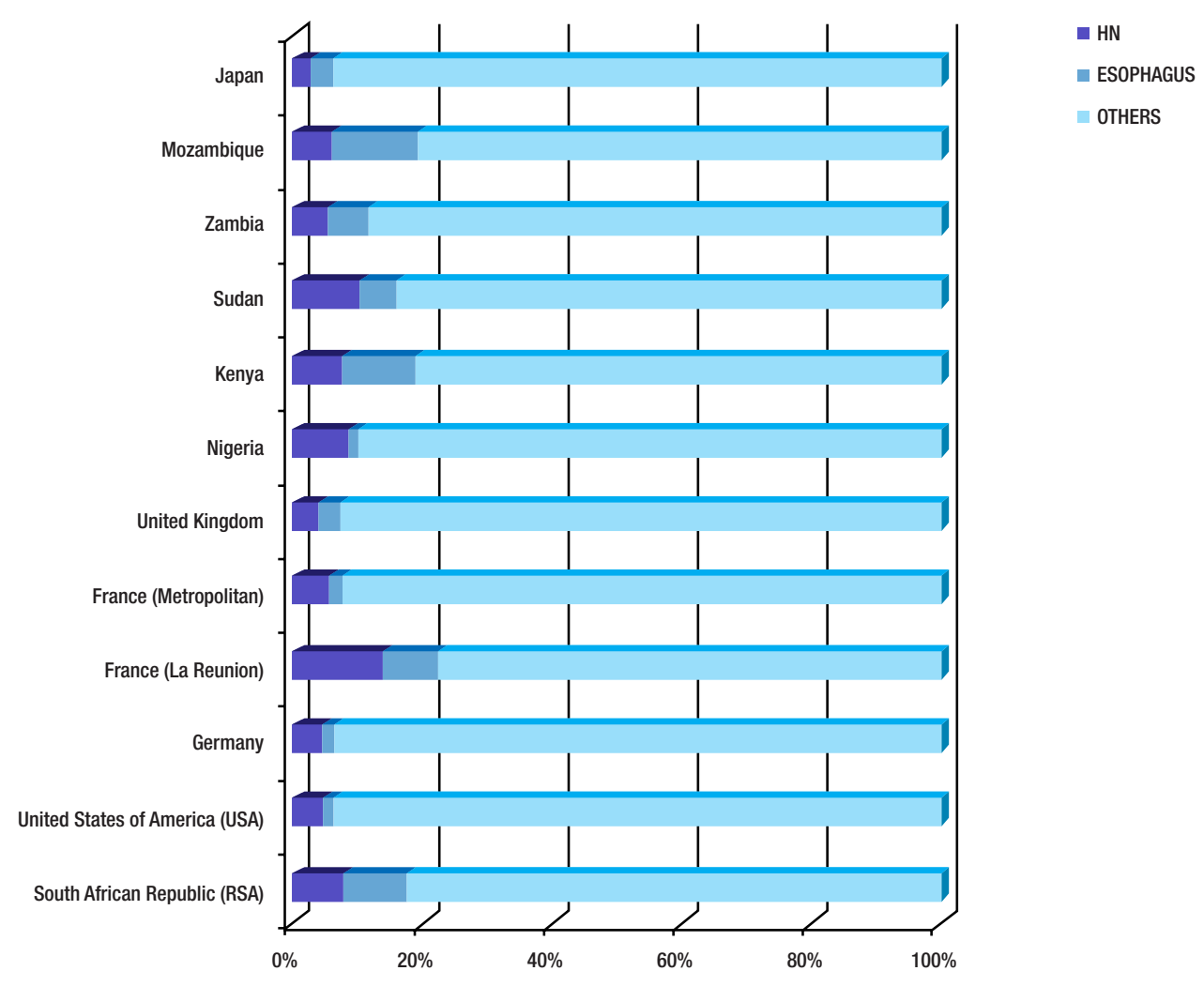

Figure 3. Stacked bar charts of the distribution of HNC and UADTC. In each bar the relative proportion of HNC (blue) and UADTC (blue and (A) Shows the high ranking distribution of HNC and UADTC by regional burgundy) are shown in comparison with other cancers (green). distribution and (B) shows similar trends by country-derived data. 


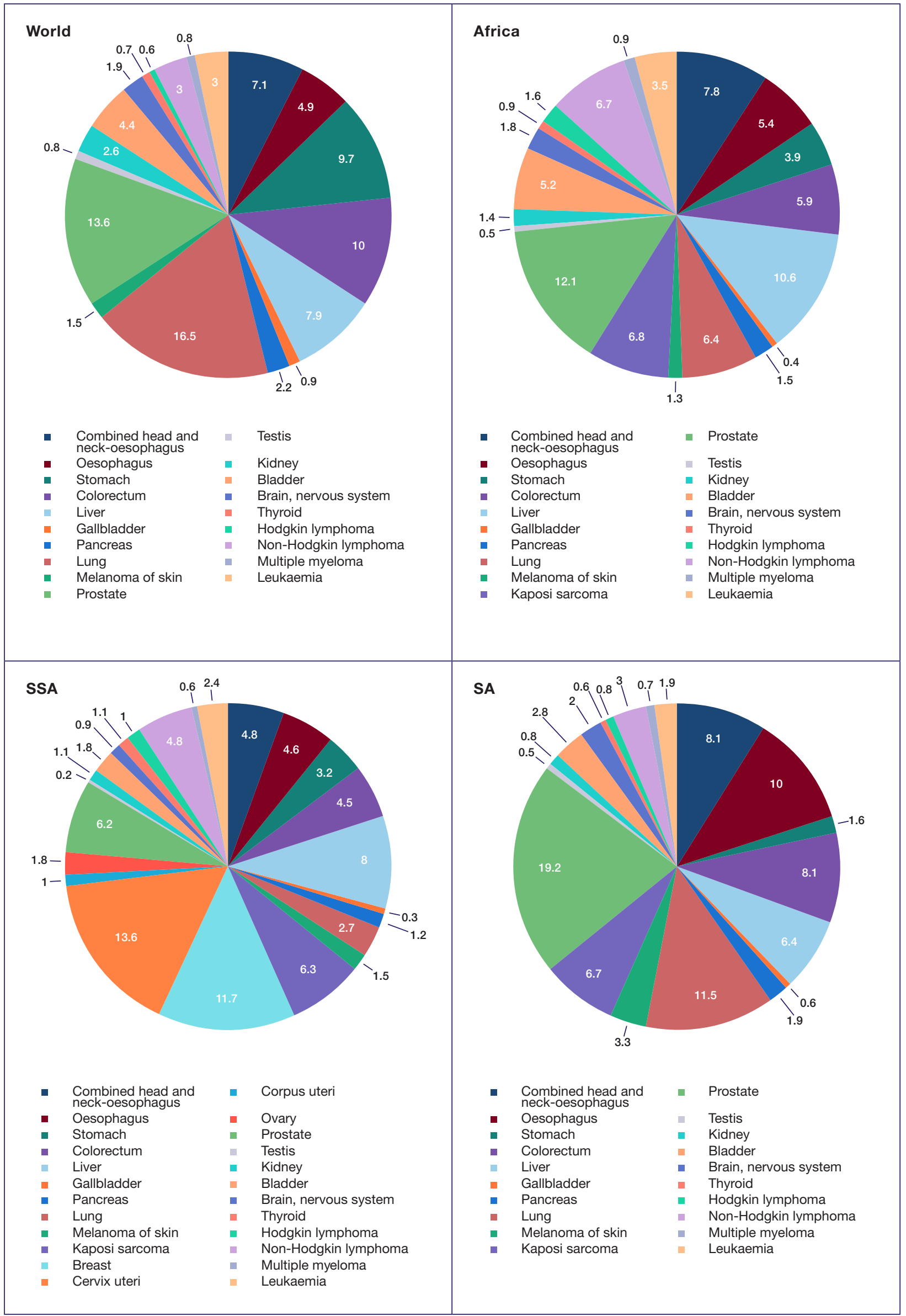

Figure 4. Pie chart showing regional distribution of HNC and UADTC in; the world, Africa, sub-Saharan (SSA) region and Southern Africa (SA). HNC (Dark blue) and UADTC (Dark blue and Burgundy) are located on the upper right quadrant of the pies. UADTC is responsible for a high fraction of cancers in Southern Africa and the burden of HNC and UADT is high in Africa as well as in the world. 


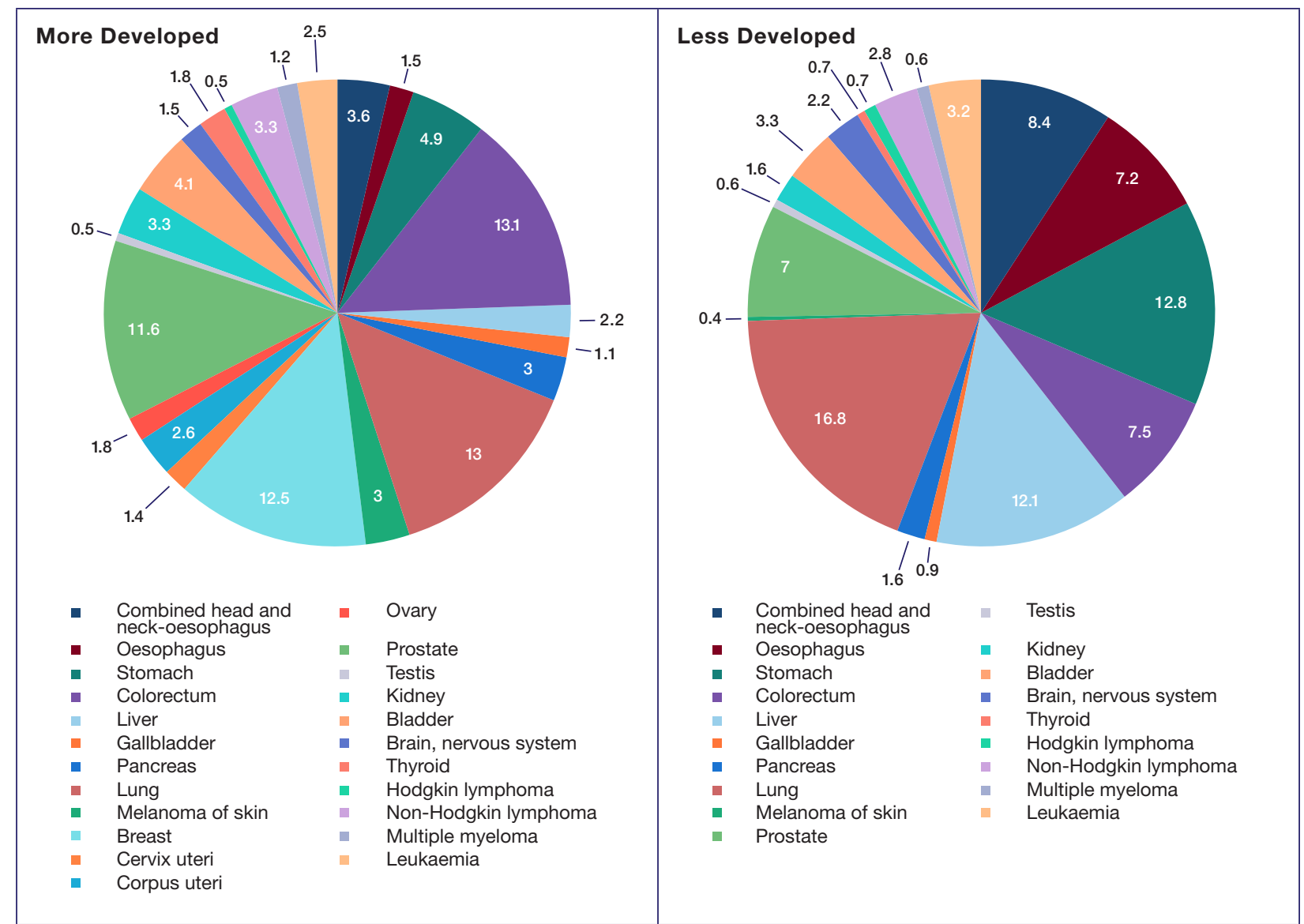

Figure 5. Pie chart of comparison of HNC and UADTC between more- and less-developed regions of the world. HNC (Dark blue) and UADTC (Dark blue and Burgundy) are located on the upper right quadrant of the pies. There is higher ranking of HNC and UADTC in the less developed regions

compared with the more developed regions. There is an almost equal distribution of $\mathrm{HNC}$ and Esophageal lesion in the less developed world; while there is a lower incidence of esophageal cancer in the more developed world compared with HNC.

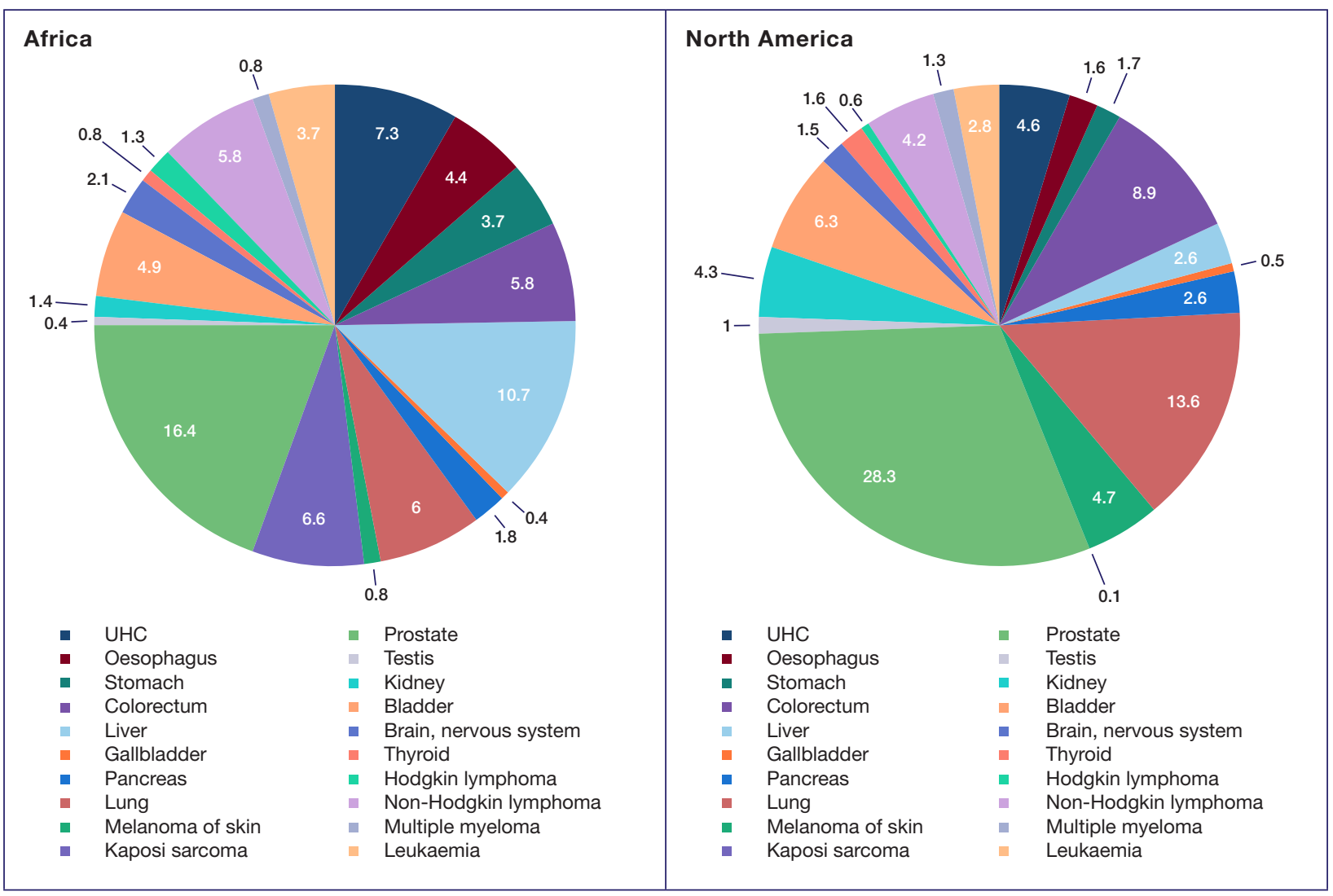

Figure 6. Pie charts showing some recent updates to the GLOBOCAN database in 2012. HNC (Dark blue) and UADTC (Dark blue and Burgundy)

are located on the upper right quadrant of the pies. HNC and UADTC still rank very high in Africa compared with North America in this new database. 


\section{DISCUSSION}

There is an increasing burden of cancer in developing economies worldwide ${ }^{2}$ and researchers in these regions may be expected to play an important part in understanding its precise impact in the regions.

With limited healthcare facilities in developing countries, there is a current struggle to attend to the burdens of various infectious diseases. They are also ill-prepared for the pandemic levels that cancer may reach if counter measures are not promptly implemented. ${ }^{22,23}$

Several factors interplay with the diagnosis and management of cancer in Africa, including cultural beliefs, religion, educational levels, lack of reliable public transport over great distances and poverty.

Religious beliefs, for example, have been found to play an important role in the attitude towards and management of cancer, ${ }^{24}$ and may play a role in a heavy cancer burden.

Political issues and governmental policies also contribute to the current situation in Africa. It has been reported that many of the poorest countries in the world allocate a substantial proportion of their health budget towards funding tertiary health facilities, as well as funding overseas health treatment trips for highly politically connected and privileged individuals, thereby denying the indigent populations access to good basic healthcare. ${ }^{25}$

Focusing on addressing the severe inequalities that exist in cancer management in LMICs will improve control in an manner analogous to HIV treatment in Africa, which many imagined was a futile effort at its outset. ${ }^{26}$

Many African countries are in the process of developing infrastructures for cancer control and management, while it appears there is minimal political will-power to support national cancer control programmes in some of the more developed economies in Africa. ${ }^{27}$

Even though the World Health Organization has developed a comprehensive cancer control programme in developing countries, it would involve a joint global effort and methodologies to facilitate its practical application. ${ }^{28}$

Importantly, beside governmental and non-governmental efforts in cancer control, an influential source of support is philanthropic ${ }^{29}$; selfless support from the public must not be underestimated. Additionally, LMICs must develop a paradigm that does not promote exclusive media hegemony in the public understanding of the cancer control agenda.

Oral cancers, as has been demonstrated, account for a significant proportion of cancers in Africa. Hence oral cancer researchers in Africa are expected to play an important role in educating the public about the increasing burden of oral cancer and possible preventive measures required to reduce such burdens.

A review of the economic burden of head and neck cancer using 77 studies, mostly from the USA, revealed a generally high cost of management. ${ }^{30}$
For example, USA spent as much as US\$8.5 billion in 2008 on cancer management; although African countries were not included in this study, such high spending would be prohibitive in Africa.

Many developing countries continue to spend small proportions of their annual gross domestic product (GDP) on healthcare. For instance, India (with a very high burden of oral cancer) in 2010 spent $3.7 \%$ of its GDP on health leading to contribution of up to $71.8 \%$ of expenditure on health from private pockets.

In contrast to this, in the United Kingdom in the same year, private contributions to healthcare expenditure totalled only $16.8 \% .{ }^{31}$ Socioeconomic considerations, illiteracy and possibly chronic infection have been identified as important related factors for oral cancer in studies from Nigeria. ${ }^{32,33}$

Another study reviewed 27 papers which had been published in Nigeria, finding a high burden of head and neck cancer in the country, ${ }^{34}$ but there is still a need for studies that compare the ranking of HNC and UADTC with other cancers in Nigeria.

Some studies described the burden of oral cancer in Kenya as very low, ${ }^{35}$ while others noticed an unchanged frequency or pattern of distribution in this population despite major lifestyle changes and the emergence of the HIV/AID epidemic. ${ }^{36}$ The same group later reported a prominence of head and neck cancer in the population. ${ }^{37}$

The initial lower incidences previously reported in Kenya as compared with our study may be due to those researchers isolating only lip and oral cancer from the HNC or UADTC.

The burden of oesophageal cancers in Kenya is high, is mainly accounted for by oral habits such as consumption of Chang'a, mursik and busaa; tobacco snuffing, and hot tea drinks, environmental nitrosamine exposure, and poor oral health ${ }^{38}$ and probably contributed to the high ranking of UADTC in Kenya, as revealed by our study.

A review of 33 oral cancer based publications from Sudan demonstrated that oral habits such as Toombak use and high risk HPV infection may play important roles in oral cancer aetiology. ${ }^{39}$

A report from the Sudanese national population-based cancer registry in Khartoum revealed that oral cancer is the sixth highest ranked cancer in all adults older than 15 years and is commoner in males than in females in this population. ${ }^{40}$

In contrast, the present study found HNC to be ranked second and UADTC ranked first, a disparity which may be due to the combined ASIR approach applied in the study.

Also to be borne in mind is the fact that there are many data discrepancies in the population and hospital based cancer registry records, particularly in the developing world. ${ }^{41}$ Even though there seems to be a higher ranking of $\mathrm{HNC}$ and UADTC in less developed areas compared with the more developed, a study from Egypt demonstrated that 
Table 1. Table showing combined ASIR ranking of HNC and UADTC in sixteen selected regions.

\begin{tabular}{|c|c|c|c|c|c|}
\hline \multirow{2}{*}{$\begin{array}{l}\text { Cancer } \\
\text { Region }\end{array}$} & \multicolumn{2}{|c|}{ Head And Neck Cancer (HNC) } & \multirow{2}{*}{$\begin{array}{l}\text { Esophagus } \\
\text { Combined ASIR }\end{array}$} & \multicolumn{2}{|c|}{$\begin{array}{l}\text { Upper Aerodigestive Tract } \\
\text { Cancer (UADTC) }\end{array}$} \\
\hline & Ranking & Combined ASIR & & Ranking & Combined ASIR \\
\hline World & 6th & 7.1 & 4.9 & $3 r d$ & 12.0 \\
\hline Europe & 4th & 6.4 & 2.0 & 4th & 8.4 \\
\hline European Union (EU-27) & 5th & 5.6 & 1.9 & 4th & 7.5 \\
\hline Central and Eastern Europe & 4 th & 8.8 & 2.2 & 4th & 11.0 \\
\hline Western Europe & 5th & 5.0 & 2.0 & 4 th & 7.0 \\
\hline Southern Europe & 5th & 6.1 & 1.3 & 5th & 7.4 \\
\hline Northern Europe & 5th & 4.0 & 2.8 & 4 th & 6.8 \\
\hline Africa & $3 r d$ & 7.8 & 5.4 & $1 s t$ & 13.2 \\
\hline Sub-Saharan Africa & 4th & 7.4 & 6.6 & 2nd & 14.0 \\
\hline Southern Africa & 4th & 8.1 & 10.0 & 2nd & 18.1 \\
\hline Western Africa & $3 r d$ & 7.3 & 1.5 & $3 r d$ & 8.8 \\
\hline Eastern Africa & 4th & 7.5 & 10.4 & 1 st & 17.9 \\
\hline Less Developed Regions & 4th & 8.4 & 7.2 & 2 nd & 15.6 \\
\hline More Developed Regions & 6th & 3.6 & 1.5 & 4 th & 5.1 \\
\hline North America & 5th & 4.7 & 1.7 & 5th & 6.4 \\
\hline Asia & 5th & 8.0 & 7.6 & $2 n d$ & 15.6 \\
\hline \multicolumn{3}{|c|}{$\begin{array}{l}\text { HNC: includes oral cavity and lip, nasopharynx, } \\
\text { other pharynx and larynx excluding the esophagus; }\end{array}$} & \multicolumn{3}{|c|}{ UADTC: includes HN + Esophagus. } \\
\hline
\end{tabular}

Table 2. Table showing relative incidence ranking of HNC and UADTC in twelve selected countries.

\begin{tabular}{|c|c|c|c|c|c|}
\hline \multirow{2}{*}{$\begin{array}{l}\text { Cancer } \\
\text { Countries }\end{array}$} & \multicolumn{2}{|c|}{ Head And Neck Cancer (HNC) } & \multirow{2}{*}{$\begin{array}{l}\text { Esophagus } \\
\text { Combined ASIR }\end{array}$} & \multicolumn{2}{|c|}{$\begin{array}{l}\text { Upper Aerodigestive Tract } \\
\text { Cancer (UADTC) }\end{array}$} \\
\hline & Ranking & Combined ASIR & & Ranking & Combined ASIR \\
\hline South African Republic (RSA) & 4th & 8.0 & 10.0 & 2nd & 18.0 \\
\hline United States Of America (USA) & 6th & 4.7 & 1.7 & 5th & 6.4 \\
\hline Germany & 5 th & 4.6 & 1.9 & 5 th & 6.5 \\
\hline France (La Reunion) & 2nd & 13.9 & 8.6 & $1 s t$ & 22.5 \\
\hline France (Metropolitan) & 4 th & 5.9 & 1.8 & 4 th & 7.7 \\
\hline United Kingdom & 5th & 4.0 & 3.5 & 4th & 7.5 \\
\hline Nigeria & $3 r d$ & 8.9 & 1.4 & 3rd & 10.3 \\
\hline Zambia & 4th & 5.5 & 6.4 & 3rd & 10.3 \\
\hline Kenya & $3 r d$ & 7.7 & 11.2 & 1st & 18.9 \\
\hline Mozambique & 5 th & 6.1 & 13.4 & 3rd & 19.5 \\
\hline Sudan & 2nd & 10.5 & 5.7 & $1 \mathrm{st}$ & 16.2 \\
\hline Japan & 9th & 3.1 & 4.1 & 5th & 7.2 \\
\hline \multicolumn{3}{|c|}{$\begin{array}{l}\text { HNC: includes oral cavity and lip, nasopharynx, other pharynx and larynx } \\
\text { excluding the esophagus; }\end{array}$} & \multicolumn{3}{|c|}{ UADTC: includes HN + Esophagus. } \\
\hline
\end{tabular}


the overall incidence of HNC was higher in the urban areas as compared with the rural areas. ${ }^{42} \mathrm{An}$ increasing trend in the incidence of HNC in South Africa has been reported over the years from 1992 to $2001,{ }^{43}$ and it was observed that Mixed Ancestry ("Coloured") South Africans had the highest incidences among all ethnicities.

It was also observed that Blacks had the least incidences, but this is almost certainly due to poor access to healthcare, especially in the rural areas, leading to missing data.

Such intra-country disparity in HNC and UADTC distribution is yet to be investigated in many African countries and warrants further research.

\section{CONCLUSION AND RECOMMENDATION}

The combined burden of cancers in the head and neck region requires substantial further research attention in Africa. Understanding the molecular biology and potential novel targeted therapies ${ }^{44-49}$ which may be applicable for oral cancer in Africans will bring appreciable progress to reducing the burden of HNC and UADTC in Africa.

Considering that the diagnostic and treatment opportunities for HNC and UADTC in sub-Saharan Africa are generally dismal, there is a great need to prioritise the financing of UADTC research efforts in Africa by NGO's, governmental agencies, philanthropists and health policy makers.

Establishment of mutually beneficial collaborations, linkages, training and infrastructure development should be urgently promoted in Africa through establishing sustainable Research and Education Networks (RENs) within Africa, in collaboration with the more developed world.

As the journey of understanding the true burden of HNC and UADTC in Africa unfolds; clinicians, pathologists, basic medical scientists, molecular cancer biologists and statisticians need to apply their concerted efforts to reducing the burden of cancer in the continent - and globally.

\section{Conflicts of interests}

The Authors declare that they have no conflict of financial or non-financial interest.

\section{Authors' contributions}

HAA was involved in the conceptualization/design (45\%), manuscript drafting (60\%), data analysis (50\%) and revision (40\%); AHA was involved in the design (10\%), manuscript drafting (10\%) data analysis (10\%) and revision (20\%); JJH was involved in the conceptualization/design (45\%), manuscript drafting (30\%), data analysis (40\%) and revision of this work.

All authors were involved in preparing the manuscript and had final approval of the submitted and published versions.

\section{Acknowledgements}

We appreciate the efforts of Associate Professor Jennifer Moodley of the School of Public Health and Cancer Research Initiative of the Faculty of Health Sciences,
University of Cape Town in providing preliminary peer review for this work.

HAA thanks the South African Medical Research Council (SAMRC) for a mid-career Scientist Research Fellowship.

\section{References}

1. Parkin DM, Pisani P, Ferlay J. Global cancer statistics. CA: A Cancer Journal for Clinicians. 1999;49(1):33-64.

2. Jemal A, Bray F, Center MM, Ferlay J, Ward E, Forman D. Global cancer statistics. CA: A Cancer Journal for Clinicians. 2011;61(2):69-90.

3. Torre LA, Bray F, Siegel RL, Ferlay J, Lortet-Tieulent J, Jemal A. Global cancer statistics, 2012. CA Cancer J Clin. 2015;65(2):87-108.

4. de Martel C, Ferlay J, Franceschi S, Vignat J, Bray F, Forman D, et al. Global burden of cancers attributable to infections in 2008: a review and synthetic analysis. The Lancet Oncology. 2012;13(6):607-15.

5. Ferlay J, Soerjomataram I, Dikshit R, Eser S, Mathers C, Rebelo M, et al. Cancer incidence and mortality worldwide: sources, methods and major patterns in GLOBOCAN 2012. Int J Cancer. 2015;136(5):E359-86.

6. Rivera C. Essentials of oral cancer. Int $J$ Clin Exp Pathol. 2015;8(9):11884-94.

7. Johnson N. Tobacco use and oral cancer: a global perspective. Journal of Dental Education. 2001;65(4):328-39.

8. Coelho KR. Challenges of the oral cancer burden in India. Journal of Cancer Epidemiology. 2012;2012:701932.

9. Warnakulasuriya S. Global epidemiology of oral and oropharyngeal cancer. Oral Oncology. 2009;45(4-5):309-16.

10. Glick M, Johnson NW. Oral and oropharyngeal cancer: what are the next steps? Journal of the American Dental Association. 2011;142(8):892-4.

11. Knaul FM, Atun R, Farmer P, Frenk J. Seizing the opportunity to close the cancer divide. Lancet. 2013;381(9885):2238-9.

12. Dalal S, Beunza JJ, Volmink J, Adebamowo C, Bajunirwe $F$, Njelekela $M$, et al. Non-communicable diseases in sub-Saharan Africa: what we know now. International Journal of Epidemiology. 2011;40(4):885-901.

13. Yanofsky VR, Mercer SE, Phelps RG. Histopathological variants of cutaneous squamous cell carcinoma: a review. Journal of Skin Cancer. 2011;2011:210813.

14. Ferlay J, Shin HR, Bray F, Forman D, Mathers C, Parkin DM. Estimates of worldwide burden of cancer in 2008: GLOBOCAN 2008. Int J Cancer. 2010;127(12):2893-917.

15. Argiris A, Karamouzis MV, Raben D, Ferris RL. Head and neck cancer. Lancet. 2008;371(9625):1695-709.

16. Guru K, Manoor UK, Supe SS. A comprehensive review of head and neck cancer rehabilitation: physical therapy perspectives. Indian J Palliat Care. 2012;18(2):87-97.

17. Marur S, Forastiere AA. Head and neck cancer: changing epidemiology, diagnosis, and treatment. Mayo Clin Proc. 2008;83(4):489-501.

18. Marron M, Boffetta P, Moller $H$, Ahrens W, Pohlabeln $H$, Benhamou $S$, et al. Risk of upper aerodigestive tract cancer and type of alcoholic beverage: a European multicenter case-control study. Eur J Epidemiol. 2012;27(7):499-517.

19. Wenig BM. Squamous cell carcinoma of the upper aerodigestive tract: precursors and problematic variants. Modern pathology : an official journal of the United States and Canadian Academy of Pathology, Inc. 2002;15(3):229-54.

20. Muir C, Weiland L. Upper aerodigestive tract cancers. Cancer. 1995;75(1 Suppl):147-53.

21. Bravi F, Edefonti V, Randi G, Ferraroni M, La Vecchia C, Decarli A. Dietary patterns and upper aerodigestive tract cancers: an overview and review. Ann Oncol. 2012;23(12):3024-39.

22. CanTreat I. Scaling up cancer diagnosis and treatment in developing countries: what can we learn from the HIV/AIDS epidemic? Ann Oncol. 2010;21(4):680-2. 
23. Jemal A, Bray F, Forman D, O'Brien M, Ferlay J, Center M, et al. Cancer burden in Africa and opportunities for prevention. Cancer. 2012;118(18):4372-84.

24. Peteet JR, Balboni MJ. Spirituality and religion in oncology. CA: a Cancer Journal for Clinicians. 2013;63(4):280-9.

25. Brock DW, Wikler D. Ethical Issues in Resource Allocation, Research, and New Product Development. In: Jamison DT, Breman JG, Measham AR, Alleyne G, Claeson M, Evans DB, et al., editors. Disease Control Priorities in Developing Countries. 2nd ed. Washington (DC)2006.

26. Farmer P, Frenk J, Knaul FM, Shulman LN, Alleyne G, Armstrong $L$, et al. Expansion of cancer care and control in countries of low and middle income: a call to action. Lancet. 2010;376(9747):1186-93.

27. Stefan DC, Elzawawy AM, Khaled HM, Ntaganda F, Asiimwe A, Addai BW, et al. Developing cancer control plans in Africa: examples from five countries. The Lancet Oncology. 2013;14(4):e189-95.

28. Ngoma T. World Health Organization cancer priorities in developing countries. Ann Oncol. 2006;17 Suppl 8:viii9-viii14.

29. Eckhouse S, Lewison G, Sullivan R. Trends in the global funding and activity of cancer research. Molecular Oncology. 2008;2(1):20-32.

30. Wissinger E, Griebsch I, Lungershausen J, Foster T, Pashos CL. The economic burden of head and neck cancer: a systematic literature review. PharmacoEconomics. 2014;32(9):865-82.

31. Joshi P, Dutta S, Chaturvedi P, Nair S. Head and neck cancers in developing countries. Rambam Maimonides Medical Journal. 2014;5(2):e0009.

32. Oji C, Chukwuneke FN. Oral cancer in Enugu, Nigeria, 1998-2003. The British Journal of Oral and Maxillofacial Surgery. 2007;45(4):298-301.

33. Lawal A, Kolude B, Adeyemi BF, Lawoyin J, Akang E. Social profile and habits of oral cancer patients in Ibadan. African Journal of Medicine and Medical Sciences. 2011;40(3):247-51.

34. da Lilly-Tariah OB, Somefun AO, Adeyemo WL. Current evidence on the burden of head and neck cancers in Nigeria. Head and Neck Oncology. 2009;1:14.

35. Kaimenyi JT. Oral health in Kenya. International Dental Journal. 2004;54(6 Suppl 1):378-82.

36. Onyango JF, Omondi BI, Njiru A, Awange OO. Oral cancer at Kenyatta National Hospital, Nairobi. East African Medical Journal. 2004;81(6):318-21.

37. Onyango JF, Awange DO, Njiru A, Macharia IM. Pattern of occurrence of head and neck cancer presenting at Kenyatta National Hospital, Nairobi. East African Medical Journal. 2006;83(5):288-91.
38. Patel K, Wakhisi J, Mining S, Mwangi A, Patel R. Esophageal Cancer, the topmost cancer at MTRH in the Rift Valley, Kenya, and its potential risk factors. ISRN Oncol. 2013;2013:503249.

39. Ahmed HG. Aetiology of oral cancer in the Sudan. Journal of Oral and Maxillofacial Research. 2013;4(2):e3.

40. Saeed IE, Weng HY, Mohamed KH, Mohammed SI. Cancer incidence in Khartoum, Sudan: first results from the Cancer Registry, 2009-2010. Cancer Medicine. 2014;3(4):1075-84.

41. Johnson NW, Jayasekara P, Amarasinghe AA. Squamous cell carcinoma and precursor lesions of the oral cavity: epidemiology and aetiology. Periodontology 2000. 2011;57(1):19-37.

42. Attar E, Dey S, Hablas A, Seifeldin IA, Ramadan M, Rozek LS, et al. Head and neck cancer in a developing country: a population-based perspective across 8 years. Oral Oncology. 2010;46(8):591-6.

43. Ayo-Yusuf OA, Lalloo R, Johnson NW. Trends and ethnic disparities in oral and oro-pharyngeal cancers in South Africa, 1992-2001. Journal of the South African Dental Association 2013;68(4):168-73.

44. Matta A, Ralhan R. Overview of current and future biologically based targeted therapies in head and neck squamous cell carcinoma. Head and Neck Oncology. 2009;1:6.

45. Bhaijee F, Pepper DJ, Pitman KT, Bell D. New developments in the molecular pathogenesis of head and neck tumors: a review of tumor-specific fusion oncogenes in mucoepidermoid carcinoma, adenoid cystic carcinoma, and NUT midline carcinoma. Annals of Diagnostic Pathology. 2011;15(1):69-77.

46. Hunt JL. An update on molecular diagnostics of squamous and salivary gland tumors of the head and neck. Archives of Pathology and Laboratory Medicine. 2011;135(5):602-9.

47. Park BJ, Chiosea SI, Grandis JR. Molecular changes in the multistage pathogenesis of head and neck cancer. Cancer Biomarkers : section A of Disease Markers. 2010;9(1-6):325-39.

48. Scully C, Bagan J. Oral squamous cell carcinoma: overview of current understanding of aetiopathogenesis and clinical implications. Oral Diseases. 2009;15(6):388-99.

49. St John MA, Li Y, Zhou X, Denny P, Ho CM, Montemagno C, et al. Interleukin 6 and interleukin 8 as potential biomarkers for oral cavity and oropharyngeal squamous cell carcinoma. Archives of Otolaryngology--Head and Neck Surgery. 2004;130(8):929-35.

\section{NOTICE OF 19th ANNUAL GENERAL MEETING (AGM) OF The South African Dental Association NPC ("SADA")}

Notice is hereby given that the 19th Annual General Meeting (AGM) of the South African Dental Association (SADA) will be held at the SADA Head Office, 31 Princess of Wales Terrace, Parktown, Johannesburg on Thursday 14 March 2019 at 18:00 followed by snacks and refreshments.

The Agenda for the meeting will be posted on the SADA website.

SADA is your association and your voice counts.

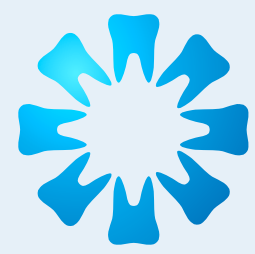

SADA

THE SOUTH AFRICAN DENTAL ASSOCIATION

\section{KC Makhubele}

Chief Executive Officer

28 September 2018 